\title{
A 2-approximation for the maximum satisfying bisection problem ${ }^{\text {is }}$
}

\author{
Bernard Ries ${ }^{\mathrm{a}, *}$, Rico Zenklusen ${ }^{\mathrm{b}}$ \\ ${ }^{a}$ LAMSADE, Université Paris Dauphine, France \\ ${ }^{\mathrm{b}}$ Department of Mathematics, MIT, USA \\ Partially supported by the Swiss National Science Foundation, grant number: \\ PBEZP2-129524. Partially developed while working at EPFL, and while visiting the \\ University of Warwick.
}

E-mail addresses: bernard.ries@dauphine.fr (B. Ries), ricoz@math.mit.edu (R. Zenklusen).

\begin{abstract}
A B S T R A C T
Given a graph $G=(V, E)$, a satisfying bisection of $G$ is a partition of the vertex set $V$ into two sets $V_{1}, V_{2}$, such that $\left|V_{1}\right|=\left|V_{2}\right|$, and such that every vertex $v \in V$ has at least as many neighbors in its own set as in the other set. The problem of deciding whether a graph $G$ admits such a partition is $\mathcal{N} \mathcal{P}$-complete. In Bazgan et al. (2008) [C. Bazgan, Z. Tuza, D. Vanderpooten, Approximation of satisfactory bisection problems, Journal of Computer and System Sciences 75 (5) (2008) 875-883], the authors present a polynomial-time 3 -approximation for maximizing the number of satisfied vertices in a bisection. It remained an open problem whether one could find a $(3-c)$-approximation, for $c>0$ (see Bazgan et al. (2010) [C. Bazgan, Z. Tuza, D. Vanderpooten, Satisfactory graph partition, variants, and generalizations, European Journal of Operational Research 206 (2) (2010) 271-280]). In this paper, we solve this problem by presenting a polynomial-time 2-approximation algorithm for the maximum number of satisfied vertices in a satisfying bisection.
\end{abstract}

\section{Introduction}

In [4,5], the authors introduced the following problem. Given a graph $G$, decide whether its vertex set $V$ can be partitioned into two nonempty sets such that each vertex has at least as many neighbors in the set it belongs to as in the other set (such a vertex will be called satisfied). Such a partition is called a satisfactory partition. Not all graphs admit such a partition. Indeed, the complete graphs $K_{n}$ for instance or the complete bipartite graphs $K_{n_{1}, n_{2}}$, with $n_{1}$ and $n_{2}$ both odd, do not admit a satisfactory partition. Several variants of the satisfactory partition problem have been defined. One of them asks for deciding whether the vertex set of a given graph can be partitioned into two nonempty sets such that each vertex has at least as many neighbors in the set it does not belong to as in its own set. Such a partition is referred to as a co-satisfactory partition or an unfriendly partition. Any finite graph admits such a co-satisfactory partition. In fact, such a partition can simply be obtained by starting with any partition of the vertices and iteratively moving a vertex that is not co-satisfied to the opposite partition. Many other variants of the satisfactory partition problem have been defined. For a current state-of-art, the reader is referred to a recent survey [3].
Satisfactory partitions as well as co-satisfactory partitions have various applications which occur in different contexts. Identifying communities in social networks, creating alliances or coalitions are some examples (see [3] for more applications). Notice that in the satisfactory partition problem (resp. co-satisfactory partition problem), the sizes of the sets of the partition are arbitrary (but different from zero). In fact, for many of the graphs for which it has been shown that a satisfactory partition exists, the difference between the sizes of the partition sets is generally very large (see for instance [2]). In the case of a 4-regular graph $G$ for example, one can always find a satisfactory partition $V_{1}, V_{2}$ of $V$ that satisfies $\left|V_{1}\right| \leqslant 5$ or $V_{1}=V(C)$, where $C$ is a shortest cycle in $G$ (see [2]). In many of the applications though, one is typically interested in finding partition sets which have mo re or less equal size. If we impose that both sets have exactly the same size, we get the so called (co-)satisfactory bisection problem.

All above mentioned problems (except the co-satisfactory problem) have been shown to be $\mathcal{N P}$-complete, and thus it is natural to look for good approximation algorithms. In [2], the authors present a polynomial-time 3-approximation algorithm for the maximum number of satisfied vertices in a bisection. In [3], a list of open problems related to satisfactory graph partitions is given. Among these problems was the following.

Design a polynomial-time $(3-c)$-approximation for the maximum number of satisfied vertices in a bisection (for as large $\mathrm{c}>0$ as possible).

After introducing some basic notations and definitions in Section 2, we present in Section 3 a polynomial-time 2-approximation algorithm for the maximum number of satisfied vertices in a 
satisfying bisection, and thus solve the above mentioned open problem for $c=1$.

\section{Preliminaries}

All graphs in this paper are finite, undirected, without loops or multiple edges. For any graph theoretical terms not defined here, the reader is referred to [8].

For a graph $G$, we denote by $V$ and $E$ the vertex set and the edge set of $G$, respectively. An edge joining two vertices $u$ and $v$ is denoted by $u v$. For a subset $U \subseteq V$ and a vertex $v \in V$, we denote by $d_{U}(v)$ the number of neighbors of $v$ in $U$. The degree of $v$ in $G$ is denoted by $d(v)$ and the set of neighbors of a vertex $v$ in $G$ is denoted by $N(v)$. The subgraph of $G$ induced by a set of vertices $U \subseteq V$ is denoted by $G[U]$.

A cut-vertex of a graph $G$ is a vertex whose deletion increases the number of connected components of $G$. We write $G-v$ (resp. $G+v$ ) for the subgraph obtained by deleting (resp. adding) a vertex $v$. Similarly, we denote by $V-v$ (resp. $V+v$ ) the subset of vertices in $G$ obtained by deleting (resp. adding) a vertex $v$. A block of a graph $G$ is a maximal connected subgraph of $G$ that has no cutvertex. Let $G\left[B_{1}\right], \ldots, G\left[B_{q}\right]$ be the blocks of $G$, where $B_{1}, \ldots, B_{q} \subseteq V$. We call the collection $\mathcal{B}=\left\{B_{1}, B_{2}, \ldots, B_{q}\right\}$ the block decomposition of $G$. It is known that a graph $G$ that is not a single block has at least two blocks, called leaf-blocks, which contain each exactly one cutvertex of $G$ (see [8]). With a slight abuse of terminology, we will call a vertex-set $B_{i} \subseteq V$ a block of $G$ if $G\left[B_{i}\right]$ is a block of $G$.

In our approach, we will use the same helpful tool as the authors of [2], that is, the Edmonds-Gallai decomposition of a graph. Before defining this type of decomposition, let us first give some definitions concerning matchings.

A matching $M$ is a set of pairwise non-adjacent edges. Let $M$ be a matching of a graph $G$. The vertices of $G$ incident to edges of $M$ are said to be saturated by $M$; the other vertices are said to be unsaturated by $M$. A perfect matching in a graph $G$ is a matching that saturates every vertex of $G$. A graph $G$ is factor-critical, if for each $v \in V$, $G-v$ admits a perfect matching. Given a matching $M$, an alternating path with respect to $M$ is a path which alternates between edges in $M$ and edges not in $M$.

For a graph $G=(V, E)$ let us denote the Edmonds-Gallai decomposition by $(X, Y, W)$, where $X, Y, W$ form a partition of $V$ that satisfies the following conditions. The set $X$ is defined such that for every vertex $v \in X$, the size of a maximum cardinality matching in $G-v$ and $G$ is the same. The set $Y$ contains all neighbors of $X$ in $V X$, and $W=V \backslash(X \cup Y)$. For more information on the EdmondsGallai decomposition see for example [6].

In particular, classical results on the Edmonds-Gallai decomposition imply that every component of $G[X]$ is factor-critical, every component of $G[W]$ admits a perfect matching and furthermore the size of a maximum cardinality matching in $G$ is equal to the number of connected components of $G[X]$ minus $|Y|$.

Consider a maximization problem $A$ and an instance $I$ of $A$. Let $S$ be a solution of $I$. We denote by $f(I, S)$ the value of solution $S$, and by $O P T(I)$ the value of an optimal solution of $I$. Then an algorithm is said to be a $k$-approximation algorithm for problem $A$, where $k \geqslant 1$, if for any instance $I$ of the problem it gives a solution $S$ such that $f(I, S) \geqslant \frac{O P T(I)}{k}$.

Let $G=(V, E)$ be a graph. A partition $V_{1}, V_{2}$ of $V$ is said to be nontrivial if both sets $V_{1}$ and $V_{2}$ are nonempty. A partition is called balanced if $\left|V_{1}\right|-\left|V_{2}\right| \in\{-1,0,1\}$, and it is called a bisection if $\left|V_{1}\right|=\left|V_{2}\right|$.

In this paper we will be interested in an optimization version of the following problem.

Input: A graph $G=(V, E)$.
Question: Does there exist a bisection $\left(V_{1}, V_{2}\right)$ of $V$ such that for every vertex $v \in V$, if $v \in V_{i}$ (for $\left.i=1,2\right)$ then $d_{V_{i}}(v) \geqslant\left\lceil\frac{d(v)}{2}\right\rceil$ ?

A vertex $v \in V$ such that if $v \in V_{i}($ for $i \in\{1,2\})$ then $d_{V_{i}}(v) \geqslant\left\lceil\frac{d(v)}{2}\right\rceil$ is called satisfied in $G$ (with respect to the partition $V_{1}, V_{2}$ ). In [1], the authors showed that the SATISFACTORY BISECTION PROBLEM is $\mathcal{N P}$-complete. In [2], the same authors presented a polynomialtime 3-approximation algorithm for the following problem.

\section{MAX SATISFYING BISECTION PROBLEM}

Input: A graph $G=(V, E)$ with $|V|$ even

Question: A bisection $\left(V_{1}, V_{2}\right)$ of $V$ that maximizes the number of satisfied vertices.

In Section 3 we will present a polynomial-time 2-approximation algorithm for this problem, thus settling an open problem mentioned in [3].

\section{2-Approximation}

In order to get a 2-approximation algorithm for the MAX SATISFYING BISECTION PROBLEM in a graph $G$, we will work in the complement of $G$, denoted by $G^{c}$. To translate the problem into $G^{c}$, we introduce the following definition.

Definition 3.1 (strong co-satisfaction). Let $G=(V, E)$ be a graph and let $V_{1}, V_{2}$ be a partition of $V$. A vertex $v \in V$ is called strongly cosatisfied (with respect to the partition $V_{1}, V_{2}$ ) if strictly more than half of the neighbors of $v$ are not in the same partition as $v$, i.e., if $v \in V_{i}$ then $\left|N(v) \cap V_{3-i}\right|>\frac{d(v)}{2}$, for $i=1,2$.

The following proposition shows how the MAX SATISFYING BISECTION PROBLEM in $G$ can be restated in the complement graph $G^{c}$

Proposition 3.2. Let $G=(V, E)$ be a graph with an even number of vertices, and let $V_{1}, V_{2}$ be a bisection of $V$. A vertex is satisfied in $G$ (with respect to the partition $V_{1}, V_{2}$ ) if and only if it is strongly co-satisfied in the complement of $G$ with respect to the same partition $V_{1}, V_{2}$.

Proof. Let $G=(V, E)$ be a graph with an even number of vertices and let $V_{1}, V_{2}$ be a bisection of $V$. Consider a vertex $v \in V_{1}$ (the case $v \in V_{2}$ is analogous). Then $v$ is satisfied in $G$ if and only if $\left|V_{2} \backslash N(v)\right| \geqslant\left|V_{1} \backslash(N(v)+v)\right|$. Since $|V|$ is even, this inequality must be strict. Since the roles of neighbors and non-neighbors are exchanged in the complement $G^{c}$ of $G$, this is equivalent to say that in $G^{c}$ the vertex $v$ has strictly more neighbors in $V_{2}$ than neighbors in $V_{1}$ and is hence strongly co-satisfied in $G^{c}$.

Due to the above proposition it is natural to define the following problem.

\section{MAX STRONG CO-SATISFYING BISECTION PROBLEM}

Input: A graph $G=(V, E)$ with $|V|$ even.

Question: A bisection $\left(V_{1}, V_{2}\right)$ of $V$ that maximizes the number of strongly co-satisfied vertices.

In what follows, we present a polynomial-time 2-approximation for the MAX STRONG CO-SATISFYING BISECTION PROBLEM. By the above proposition, taking the complement $G^{c}$ of a given graph $G$ and applying this algorithm to $G^{c}$ leads to a 2-approximation for the MAX SATISFYING BISECTION PROBLEM in $G$.

Notice that the complement $G^{c}$ of a graph $G$ might be disconnected. Thus we will work on each connected component of $G^{c}$ separately. 
Definition 3.3 (edge-flip). Let $G=(V, E)$ be a graph. We say that we perform an edge-flip on an edge $u v \in E$ with respect to a partition $V_{1}, V_{2}$ of $V$, if with probability $\frac{1}{2}$ we assign $u$ to $V_{1}$ and $v$ to $V_{2}$ and with probability $\frac{1}{2}$ we assign $u$ to $V_{2}$ and $v$ to $V_{1}$.

Edge-flips can be used in a variety of settings to strongly co-satisfy half of the vertices. The following proposition shows that every time two vertices connected by an edge are randomly assigned by an edge-flip to $V_{1}, V_{2}$, in expectation at least one of these two vertices will be strongly co-satisfied.

Proposition 3.4. Let $G=(V, E)$ be a graph, let $u v \in E$, and let $V_{1}^{\prime}, V_{2}^{\prime}$ be a partition of $V\{u, v\}$. Let $V_{1}, V_{2}$ be a random partition obtained by completing $V_{1}^{\prime}, V_{2}^{\prime}$ with an edge-flip on $u v$. Then in expectation at least one of the vertices $u$ and $v$ is strongly co-satisfied with respect to the partition $V_{1}, V_{2}$.

Proof. Consider the vertex $v$ (the proof for the vertex $u$ is analogous) and assume without loss of generality that $\left|N(v) \cap V_{1}^{\prime}\right| \geqslant$ $\left|N(v) \cap V_{2}^{\prime}\right|$. Hence, if the outcome of the edge-flip on $u v$ assigns $v$ to $V_{2}$, then $v$ is strongly co-satisfied since in this case we have

$$
\begin{aligned}
\left|N(v) \cap V_{1}\right| & =\left|N(v) \cap\left(V_{1}^{\prime}+u\right)\right|=1+\left|N(v) \cap V_{1}^{\prime}\right|>\left|N(v) \cap V_{2}^{\prime}\right| \\
& =\left|N(v) \cap V_{2}\right| .
\end{aligned}
$$

For a vertex $w \in V$, let $\mathbf{1}_{w}$ be the random variable which is equal to one if $w$ is strongly co-satisfied and zero otherwise. Hence by the above reasoning, we have $\mathbf{E}\left[\mathbf{1}_{v}\right] \geqslant 1 / 2$ and $\mathbf{E}\left[\mathbf{1}_{u}\right] \geqslant 1 / 2$, and hence the expected number of strongly co-satisfied vertices among $u$ and $v$ satisfies $\mathbf{E}\left[\mathbf{1}_{u}+\mathbf{1}_{v}\right] \geqslant 1$.

The following theorem shows one setting in which the above proposition can be used to strongly co-satisfy at least half of the total number of vertices. It nicely illustrates how the reasoning based on randomization can be used to obtain results of this type. An important feature of the randomization-based approach is that to compute the expected number of strongly co-satisfied vertices, each vertex can be considered independently because of the linearity of the expectation. Due to this fact, this proof strategy is quite flexible, and can be adapted to other settings as we will see later.

Theorem 3.5. Let $G=(V, E)$ be a graph admitting a perfect matching. Then there is a polynomial-time algorithm to find a bisection of $V$ that strongly co-satisfies at least $|V| / 2$ vertices of $G$.

Proof. Let $M$ be a perfect matching in $G$. Consider a random bisection obtained by independently performing edge-flips on all edges of $M$, i.e., we start with $V_{1}, V_{2}=\emptyset$ and for every edge $u v \in M$, with probability $\frac{1}{2}$ we set $V_{1}:=V_{1}+u, \quad V_{2}:=V_{2}+v$ or otherwise $V_{1}:=V_{1}+v, V_{2}:=V_{2}+u$. For a vertex $v \in V$, again we denote by $\mathbf{1}_{v}$ the indicator variable for the event that $v$ is strongly co-satisfied.

Consider an edge $u v \in M$. By Proposition 3.4, for any bisection $V_{1}^{\prime}, V_{2}^{\prime}$ of $V-\{u, v\}$, when completing $V_{1}^{\prime}, V_{2}^{\prime}$ by performing an edge-flip on $u v$, in expectation the number of strongly co-satisfied vertices among $u, v$ is at least one. Hence, $\mathbf{E}\left[\mathbf{1}_{v}+\mathbf{1}_{u}\right] \geqslant 1$ and the expected number of strongly co-satisfied vertices satisfies $\mathbf{E}\left[\sum_{w \in V} \mathbf{1}_{w}\right]=\mathbf{E}\left[\sum_{x y \in M}\left(\mathbf{1}_{x}+\mathbf{1}_{y}\right)\right] \geqslant|M|=|V| / 2$.

The random bisection procedure can easily be derandomized using conditional expectations (see [7] for information on derandomization by conditional expectations). For completeness we shortly discuss one step of the derandomization procedure. This allows us to present the type of conditional expectations that have to be calculated to perform the derandomization, and to show why this can be done in polynomial time.
Let $M=\left\{u_{1} v_{1}, \ldots, u_{n / 2} v_{n / 2}\right\}$, where $n=|V|$. To derandomize the procedure, we assume that the edge-flips on $M$ are done iteratively. In the first step, an edge-flip on $u_{1} v_{1}$ is performed then we continue with an edge flip on $u_{2} v_{2}$ and so on. At step $k \in\{1, \ldots, n / 2\}$ of the derandomization procedure, all the vertices in $\left\{u_{1}, v_{1}, u_{2}, \ldots, v_{k-1}\right\}$ have already been assigned to either $V_{1}$ or $V_{2}$. Let $V_{1}^{k-1} \subseteq\left\{u_{1}, v_{1}\right.$, $\left.u_{2}, \ldots, v_{k-1}\right\}$ be the set containing all vertices which have already been assigned to $V_{1}$ during the iterations $1, \ldots, k-1$, and similarly we define $V_{2}^{k-1}$ to be the set of vertices that have been assigned to $V_{2}$. Hence, knowing the state just before iteration $k$, the expected number of strongly co-satisfied vertices is given by $\mathbf{E}\left[\sum_{v \in V} \mathbf{1}_{v} \mid V_{1}^{k-1}, V_{2}^{k-1}\right]=\mathbf{E}\left[\sum_{v \in V} \mathbf{1}_{v} \mid V_{1}^{k-1}\right]$. Conditioning on the outcome of iteration $k$ we get

$$
\begin{aligned}
\mathbf{E}\left[\sum_{v \in V} \mathbf{1}_{v} \mid V_{1}^{k-1}\right]= & \frac{1}{2} \underbrace{\mathbf{E}\left[\sum_{v \in V} \mathbf{1}_{v} \mid V_{1}^{k}=V_{1}^{k-1}+u_{k}\right]}_{(I)} \\
& +\frac{1}{2} \underbrace{\mathbf{E}\left[\sum_{v \in V} \mathbf{1}_{v} \mid V_{1}^{k}=V_{1}^{k-1}+v_{k}\right]}_{(I I)} .
\end{aligned}
$$

The main idea of derandomization by conditional expectations is to compare the two expectations (I) and (II). At least one of these expectations must be larger or equal than their mean which is the left-hand side of (1). If the value of $(I)$ is larger or equal to the value of $(I I)$, then, instead of randomly flipping the edge $u_{k} v_{k}$, we set $V_{1}^{k}=V_{1}^{k-1}+u_{k}$, otherwise we set $V_{1}^{k}=V_{1}^{k-1}+v_{k}$. By doing so, we are guaranteed to obtain an assignment such that $\mathbf{E}\left[\sum_{v \in V} \mathbf{1}_{v} \mid V_{1}^{k}\right] \geqslant$ $\mathbf{E}\left[\sum_{v \in V} \mathbf{1}_{v} \mid V_{1}^{k-1}\right]$.

To perform the above derandomization, we have to determine the conditional expectations (I) and (II). Consider for example (I) (the case (II) is analogous). By linearity of the expectation, $(I)$ is equal to $\sum_{v \in V} \mathbf{E}\left[\mathbf{1}_{v} \mid V_{1}^{k}\right]$. Hence, to evaluate $(I)$, it suffices to determine terms of type $\mathbf{E}\left[\mathbf{1}_{v} \mid V_{1}^{k}\right]=\operatorname{Pr}[v$ is strongly co-satisfied $\left.\mid V_{1}^{k}\right]$ for $v \in V$, i.e., given is a partial assignment of the vertices to the sets of the partition and one has to determine for a given vertex $v$ the probability that $v$ will be strongly co-satisfied. We distinguish two cases depending on whether $v$ has already been assigned to one of $V_{1}^{k}, V_{2}^{k}$, i.e., $v \in\left\{u_{1}, v_{1}, \ldots, u_{k}, v_{k}\right\}$, or not.

Case (a) $v \in V_{1}^{k} \cup V_{2}^{k}$. Let $n^{\prime}$ and $n^{\prime \prime}$, respectively, be the number of already assigned neighbors of $v$ that are in the same partition as $v$ and the opposite partition, respectively. The non-assigned neighbors $A$ of $v$ can be partitioned into two groups $A^{\prime}, A^{\prime \prime}$, where $A^{\prime}$ consists of the endpoints of all edges $e \in M \cap(A \times A)$, and $A^{\prime \prime}=A \backslash A^{\prime}$. Notice that since we are performing edge-flips on the edges in $M$, exactly half of the vertices of $A^{\prime}$ will be in the same partition as $v$. Hence, the probability of $v$ being strongly co-satisfied only depends on how the vertices in $A^{\prime \prime}$ will be assigned to the partitions. Note that every vertex in $A^{\prime \prime}$ is assigned to a partition independently of the other vertices in $A^{\prime \prime}$, since every vertex in $A^{\prime \prime}$ is incident to a different edge of $M$. Let $X$ be the random variable corresponding to the number of vertices in $A^{\prime \prime}$ that will be assigned to the opposite partition of $v$. Observe that after all edge-flips, $v$ is strongly co-satisfied if and only if $X>n^{\prime}-n^{\prime \prime}$. Since $X$ is a binomial random variable with parameters $\left|A^{\prime \prime}\right|$ and $1 / 2$, the probability $\operatorname{Pr}\left[X>n^{\prime}-n^{\prime \prime}\right]$ can easily be evaluated in polynomial time.

Case (b) $v \notin V_{1}^{k} \cup V_{2}^{k}$. We have $v=v_{j}$ for some $j \in\{k+1, \ldots, n / 2\}$. Since all edge-flips that still have to be performed are done independently we can assume $j=k+1$ by renumbering the vertices in $\left\{v_{k+1}, u_{k+1}, \ldots, v_{n / 2}, u_{n / 2}\right\}$. To evaluate the expectation $\mathbf{E}\left[\mathbf{1}_{v} \mid V_{1}^{k}\right]$, we can condition on the outcome of the next flip which involves $v$, i.e., the flip of the edge $v_{k+1} u_{k+1}$, i.e.,

$$
\mathbf{E}\left[\mathbf{1}_{v} \mid V_{1}^{k}\right]=\frac{1}{2} \mathbf{E}\left[\mathbf{1}_{v} \mid V_{1}^{k+1}=V_{1}^{k}+v_{k+1}\right]+\frac{1}{2} \mathbf{E}\left[\mathbf{1}_{v} \mid V_{1}^{k+1}=V_{1}^{k}+u_{k+1}\right] .
$$


The two expectations to the right are again of the form of case (a) and can thus be evaluated in polynomial time, leading to a polynomial-time procedure for evaluating $\mathbf{E}\left[\mathbf{1}_{v} \mid V_{1}^{k}\right]$.

Finally, by repeating the above derandomization step until all edge-flips are done, a bisection $V_{1}=V_{1}^{n / 2}, V_{2}=V_{2}^{n / 2}$ is obtained, such that the number of strongly co-satisfied vertices is lowerbounded by

$$
\mathbf{E}\left[\sum_{v \in V} \mathbf{1}_{v} \mid V_{1}\right]=\mathbf{E}\left[\sum_{v \in V} \mathbf{1}_{v} \mid V_{1}^{\mathrm{n} / 2}\right] \geqslant \mathbf{E}\left[\sum_{v \in V} \mathbf{1}_{v} \mid V_{1}^{0}\right]=\mathbf{E}\left[\sum_{v \in V} \mathbf{1}_{v}\right] \geqslant|V| / 2 .
$$

Our next theorem shows another setting in which we are able to strongly co-satisfy at least half of the total number of vertices.

Theorem 3.6. Let $G=(V, E)$ be a graph with an even number of vertices. Let $(X, Y, W)$ be an Edmonds-Gallai decomposition of $G$. If $|Y|>0$, then there is a polynomial-time algorithm to find a bisection that strongly co-satisfies at least $|V| / 2$ vertices of $G$.

Proof. Let $X_{1}, \ldots, X_{p}$ (resp. $W_{1}, \ldots, W_{q}$ ) be the partition of $X$ (resp. of $W$ ) corresponding to the connected components of $G[X]$ (resp. of $G[W]$ ). The Edmonds-Gallai decomposition satisfies $p \geqslant|Y|$ (see [6]). Furthermore, since $|V|$ is even we can deduce that $p+|Y|$ is even, because $\left|X_{i}\right|$ for $i \in\{1, \ldots, p\}$ is odd, and all components of $G[W]$ are even. In each set $X_{i}$ we fix an arbitrary vertex $v_{i} \in X_{i}$.

In the following, we construct a bisection $V_{1}, V_{2}$ satisfying the claim of the theorem. We start with $V_{1}, V_{2}=\emptyset$ and successively add vertices to $V_{1}$ and $V_{2}$. All vertices of $Y$ are assigned to one part of the partition, say $V_{1}$. Furthermore, the vertices $v_{1}, \ldots, v_{(p+|Y|) / 2}$ are included in $V_{2}$ and the vertices $v_{(p+|Y|) / 2+1}, \ldots, v_{p}$ are included in $V_{1}$. Let $V^{\prime}=V\left(Y \cup\left\{v_{1}, \ldots, v_{p}\right\}\right)$ be the vertices which have not been assigned to either partition so far. Notice that exactly half of the so far assigned vertices are in $V_{1}$ and half in $V_{2}$.

Furthermore, notice that $G\left[V^{\prime}\right]$ admits a perfect matching because of the following. $G\left[V^{\prime}\right]$ is the union of $G[W]$ and the connected components $G\left[X_{i}-v_{i}\right]$ for $i \in\{1, \ldots, p\}$. Since $(X, Y, W)$ is an Gallai-Edmonds decomposition, $G[W]$ admits a perfect matching. Furthermore, each graph of the form $G\left[X_{i}-v_{i}\right]$ also admits a perfect matching since $G\left[X_{i}\right]$ is factor-critical. Hence, let $M$ be a perfect matching in $G\left[V^{\prime}\right]$.

In the following, we consider each connected component of $G[X \cup W]$ separately and assign for each connected component the non-assigned vertices to $V_{1}$ and $V_{2}$. This is done in such a way that for every connected component of $G[X \cup W]$, exactly half of the non-assigned vertices will be assigned to $V_{1}$ and the other half to $V_{2}$. This will guarantee that at the end of the procedure we obtain a bisection. We distinguish between three types of connected components:

(i) Connected components $G\left[X_{i}\right]$ of $G[X]$, such that $v_{i} \in V_{2}$. These are the components $G\left[X_{i}\right]$ for $i \in\{1, \ldots,(p+|Y|) / 2\}$.

(ii) Connected components $G\left[X_{i}\right]$ of $G[X]$, such that $v_{i} \in V_{1}$. These are the components $G\left[X_{i}\right]$ for $i \in\{(p+|Y|) / 2+1, \ldots, p\}$.

(iii) Connected components $G\left[W_{i}\right]$, for $i=1, \ldots, q$, which all have an even number of vertices and admit a perfect matching.

Notice that since all non-assigned vertices are part of $G[X \cup W]$, the non-assigned neighbors of a non-assigned vertex $v$ are all in the same connected component as $v$. Hence, any assignment of the non-assigned vertices in one connected component of $G[X \cup W]$ does not have any influence on whether vertices in another connected component of $G[X \cup W]$ will be strongly co-satisfied or not. Hence, we can assign the non-assigned vertices of any connected component of $G[X \cup W]$ independently of the other connected components of $G[X \cup W]$. We will show how to assign in polynomial time the non-assigned vertices to $V_{1}$ and $V_{2}$ such that the following holds.

(a) In every component $G\left[X_{i}\right]$ of type (i), at least $\left(\left|X_{i}\right|+1\right) / 2$ vertices will be strongly co-satisfied.

(b) In every component $G\left[X_{i}\right]$ of type (ii), at least $\left(\left|X_{i}\right|-1\right) / 2$ vertices will be strongly co-satisfied.

(c) In every component $G\left[W_{i}\right]$ of type (iii), at least $\left|W_{i}\right| / 2$ vertices will be strongly co-satisfied.

Notice that once the three statements above are proven we are done, since the total number of strongly co-satisfied vertices is then at least

$$
\begin{aligned}
& \sum_{i=1}^{(p+|Y|) / 2}\left(\left|X_{i}\right|+1\right) / 2+\sum_{i=1+(p+|Y|) / 2}^{p}\left(\left|X_{i}\right|-1\right) / 2+|W| / 2 \\
& \quad=|X| / 2+|W| / 2+|Y| / 2=|V| / 2 .
\end{aligned}
$$

Hence it remains to prove that we can achieve (a), (b) and (c).

Proof of $(a)$ and $(b)$ : Consider a connected component $G\left[X_{i}\right]$ of $G[X]$. We will distinguish later whether we are in the case $v_{i} \in V_{2}$ (case (a)) or $v_{i} \in V_{1}$ (case (b)). Consider the procedure that assigns the non-assigned vertices in $X_{i}$ in a random way to $V_{1}$ and $V_{2}$ by performing edge-flips on the edges of $M$ in $G\left[X_{i}\right]$. By Proposition 3.4 we can deduce that in expectation, such an assignment will strongly co-satisfy at least $\left(\left|X_{i}\right|-1\right) / 2$ vertices among the vertices of $X_{i}-v_{i}$. Similarly to the proof of Theorem 3.5 this approach can be derandomized using conditional expectations to get a polynomial-time procedure to assign the vertices in $X_{i}$ such that at least $\left(\left|X_{i}\right|-1\right) / 2$ vertices in $X_{i}$ are strongly co-satisfied. This already proves part (b).

Furthermore, if $v_{i} \in V_{2}$ then there is a strictly positive probability that in the random assignment, the vertex $v_{i}$ will be strongly cosatisfied, because all neighbors of $v_{i}$ in $Y$ are in the opposite partition and every other neighbor is with probability $1 / 2$ in the opposite partition. ${ }^{1}$ Hence, in this case, the expected number of strongly co-satisfied vertices in $X_{i}$ is strictly more than $\left(\left|X_{i}\right|-1\right) / 2$. By derandomizing we obtain a polynomial-time procedure to assign the vertices in $X_{i}$ such that at least $\left(\left|X_{i}\right|+1\right) / 2$ vertices are strongly co-satisfied, proving (a).

Proof of (c): Let $G\left[W_{i}\right]$ be a connected component of $G[W]$. Again, we first consider a random bisection of $W_{i}$ obtained by performing edge-flips on the edges of $M$ in $G\left[W_{i}\right]$. As before, we observe that the expected number of strongly co-satisfied vertices is at least $\left|W_{i}\right| / 2$ and the result follows by derandomization via conditional expectations.

Theorems 3.5 and 3.6 show already how to obtain a 2-approximation for the MAX STRONG CO-SATISFYING BISECTION PROBLEM for a large class of graphs. It remains to deal with the case where we have a Gallai-Edmonds decomposition $(X, Y, W)$ of the given graph $G$ that satisfies $Y=\emptyset$. In this case $G$ consists of factor-critical connected components, i.e., the components in $G[X]$, and connected components that contain a perfect matching, i.e., the components in $G[W]$. In the following, we show how to efficiently find a balanced partition for each connected component $C$ of $G$, such that this partition strongly co-satisfies at least half of the maximum number of vertices in $V(C)$ that can be strongly co-satisfied by any (not necessarily balanced) partition of $V(C)$. Having determined such a balanced partition $V_{1}^{\prime}, V_{2}^{\prime}$ for every component $C$ of $G$, we

\footnotetext{
${ }^{1}$ In fact, one can easily show that the probability of $v_{i}$ being strongly co-satisfied is even at least 0.5 .
} 
will then put all vertices in $V_{1}^{\prime}$ in one set of the partition $V_{1}, V_{2}$ and the elements of $V_{2}^{\prime}$ in the other set, in such a way, that after having processed all components of $G$, we get $\left|V_{1}\right|=\left|V_{2}\right|$. This can easily be achieved because of the balancedness of the partition $V_{1}^{\prime}, V_{2}^{\prime}$.

The components in $G[W]$ are easy to handle, since they admit a perfect matching. Hence, we can apply Theorem 3.5 to each component $G\left[W_{i}\right]$ of $G[W]$, to find a bisection of $W_{i}$ that strongly co-satisfies at least $\left|W_{i}\right| / 2$ vertices of $W_{i}$. The factor-critical components in $G[X]$ are more challenging to find a good partition. Here we distinguish several cases. In many settings the result can again be obtained by using edge-flip techniques. More precisely, we introduce the following notion of matched neighbors property and show that for any graph not having this property, we can find a good balanced partition by edge-flips.

Definition 3.7 (matched neighbors property). We say that a factorcritical graph $G=(V, E)$ has the matched neighbors property, if for any vertex $v \in V$ and any perfect matching $M$ in $G[V-v], M$ induces a perfect matching in $N(v)$.

We first observe that it is possible to check in polynomial time whether a given graph $G$ satisfies the matched neighbors property, and if not, to find a corresponding certificate, i.e., a vertex $v \in V$ and a perfect matching $M$ in $G[V-v]$ such that $M$ does not induce a perfect matching in $N(v)$. This can be done as follows. Consider a fixed vertex $v \in V$ and a fixed neighbor $u \in N(v)$ of $v$. To check whether there is a perfect matching $M$ in $G[V-v]$ such that $u$ is not linked to another vertex in $N(v)$ by $M$, we consider the graph $G^{\prime}=(V-v, E \backslash\{u w \mid w \in N(v)\})$, and check whether $G^{\prime}$ admits a perfect matching. If this is the case, then $G$ does not satisfy the matched neighbors property, because the vertex $v$ together with the matching $M$ is a certificate therefor. Notice that $G$ satisfies the matched neighbors property if and only if for every pair of vertices $v \in V$ and $u \in N(v)$, the corresponding graph $G^{\prime}$, as defined above, does not admit a perfect matching. Hence, this can easily be checked in polynomial time.

The following proposition shows that in any graph that does not satisfy the matched neighbors property, we can find a good partition using edge-flips.

Proposition 3.8. Let $G=(V, E)$ be a factor-critical graph that does not satisfy the matched neighbors property. Then, there is a balanced partition that strongly co-satisfies at least $(|V|+1) / 2$ vertices.

Proof. Since $G$ does not satisfy the matched neighbors property, we can find a vertex $v \in V$ and a perfect matching $M$ in $G-v$ such that there is an edge $a b \in M$ with $a \in N(v)$ and $b \notin N(v)$. We put $v$ in one partition, say $V_{1}$, and perform edge-flips on all edges of $M$. Observe that there is a strictly positive probability that $v$ is strongly co-satisfied because of the following. Every neighbor of $v$ is in $V_{2}$ with probability $1 / 2$. Furthermore, the event that $a \in V_{2}$ is independent of the event that any other neighbor of $v$ is in $V_{2}$. Hence, we can conclude that with strictly positive probability more than half of the neighbors of $v$ are in $V_{2}$, in which case $v$ is strongly co-satisfied. Notice that in the above reasoning we crucially exploit the fact to have an edge $a b$ with $a \in N(v)$ and $b \notin N(v)$, since otherwise if all vertices of $N(v)$ were perfectly matched by edges of $M$, then always exactly half of the neighbors of $v$ would be in $V_{2}$, and hence, $v$ would never be strongly co-satisfied.

By Proposition 3.4 each vertex of $V-v$ is strongly co-satisfied with probability at least $1 / 2$. Hence, the expected number of strongly co-satisfied vertices is strictly more than $|V| / 2$. Using derandomization through conditional expectations, we can find in polynomial time a balanced partition strongly co-satisfying at least $\lceil|V| / 2\rceil=(|V|+1) / 2$ vertices.

To complete the discussion of factor-critical graphs, we now consider factor-critical graphs satisfying the matched neighbors property. We first observe, that by using edge-flip techniques, we can easily strongly co-satisfy $(|V|-1) / 2$ vertices in any factor critical graph.

Proposition 3.9. Let $G=(V, E)$ be a factor-critical graph. Then there is a polynomial-time algorithm to find a balanced partition strongly cosatisfying at least $(|V|-1) / 2$ vertices.

Proof. Fix an arbitrary vertex $v \in V$, and let $M$ be a perfect matching in $G[V-v]$. We associate $v$ with the first partition, i.e., $v \in V_{1}$. By Proposition 3.4, we strongly co-satisfy in expectation at least $(|V|-1) / 2$ vertices of $V-v$ by performing edge-flips on $M$. The result follows by using derandomization through conditional expectations.

A priori it is not clear whether the above procedure yields a 2approximation for the problem of finding a balanced partition that strongly co-satisfies the maximum number of vertices, since it might be possible, that there is a balanced partition strongly cosatisfying all vertices. In the following we show that if $G$ is a factor-critical graph with the matched neighbors property, then no partition strongly co-satisfies all of the vertices. This will be proven by showing that the factor-critical graphs with the matched neighbors property have a very special structure. More precisely, we will show that each block of such a graph is an odd clique. We start by showing that the matched neighbors property in a factor-critical graph holds block-wise.

Proposition 3.10. Let $G=(V, E)$ be a factor-critical graph that satisfies the matched neighbors property. Then every block of $G$ is also factorcritical and satisfies the matched neighbors property.

Proof. Let $G=(V, E)$ be a factor-critical graph that satisfies the matched neighbors property. Let $\mathcal{B}=\left\{B_{1}, B_{2}, \ldots, B_{q}\right\}$ be the block decomposition of $G$. We first claim that every leaf-block $B_{i} \in \mathcal{B}$ has odd size. Indeed, let $B_{i}$ be a leaf-block and let $w \in B_{i}$ be the unique cut-vertex of $G$ in $B_{i}$. Since $G$ is factor-critical and $G\left[B_{i}-w\right]$ is a connected component of $G-w$, it follows that any perfect matching $M$ in $G-w$ induces a perfect matching in $B_{i}-w$. Thus $B_{i}$ has odd size.

Next we claim that for every leaf-block $B_{i} \in \mathcal{B}$, the graph $G^{\prime}=$ $G\left[V\left(B_{i}-w\right)\right]$ is factor-critical and satisfies the matched neighbors property. Let $v \in V\left(B_{i}-w\right)$. Since $G$ is factor-critical, it follows that there exists a perfect matching $M$ in $G-v$. First assume that $v \neq w$. Let $w^{\prime} \in V$ such that $w w^{\prime} \in M$. Since $B_{i}$ has odd size, we conclude that $w^{\prime} \in V \backslash B_{i}$. Thus $M$ induces a perfect matching in $G^{\prime}-v$, and hence $G^{\prime}$ is factor-critical. Now assume that $v=w$. Then clearly $M$ induces a perfect matching in $G^{\prime}-v$, since $G^{\prime}-v$ is a connected component of $G-v$. Thus $G^{\prime}$ is factor-critical.

Furthermore, since $G$ has the matched neighbors property, $M$ induces a perfect matching in $N(v) \cap V$. If $w \neq v$, then $N(v) \cap V=N(v) \cap V\left(G^{\prime}\right)$ and we are done. If $w=v$, let $N_{i}(v)=N(v) \cap B_{i}$ and $N^{\prime}(v)=(N(v) \cap V) \backslash N_{i}(v)$. Since $M$ induces a perfect matching in $N(v) \cap V$, and there are no edges between $N_{i}(v)$ and $N^{\prime}(v)$, it follows that $M$ induces a perfect matching in $N^{\prime}(v)$. Hence, we conclude that $G^{\prime}$ has the matched neighbors property.

Since every block $B \in \mathcal{B}$ can be obtained from $G$ by successively deleting leaf blocks (without the corresponding cut-vertices), it follows from the above that every block in $G$ is factor-critical and satisfies the matched neighbors property. 
Proposition 3.11. Let $G=(V, E)$ be a factor-critical graph satisfying the matched neighbors property. Then either $G$ is a complete graph or G has a cut-vertex.

Proof. We assume by contradiction that $G$ is not a complete graph and that $G$ has no cut-vertex. Thus there exists a vertex $v \in V$ which has a non-neighbor. We consider the partition of $G-v$ given by $N=N(v)$ and $\bar{N}=V \backslash(N(v)+v)$. Since $G$ is connected (this follows from the fact that $G$ is factor-critical) and since $v$ has a non-neighbor, it follows that $N, \bar{N} \neq \emptyset$. Also, from the connectivity of $G$, it follows that there is a vertex $u \in N$ which is adjacent to some vertex $w \in \bar{N}$. Since $G$ is a factor-critical graph which satisfies the matched neighbors property, it follows that there exists a perfect matching $M$ in $G[V-v]$ which induces a perfect matching in $N$ and a perfect matching in $\bar{N}$. Let us denote by $u^{\prime} \in N$ and $w^{\prime} \in \bar{N}$ the vertices such that $u u^{\prime}, w w^{\prime} \in M$. We claim that $u w^{\prime} \in E$ and $u^{\prime} w, u^{\prime} w^{\prime} \notin E$. Indeed, if $u w^{\prime} \notin E$, then $M^{\prime}=\left(M \backslash\left\{u u^{\prime}\right\}\right) \cup\left\{v u^{\prime}\right\}$ is a perfect matching in $G-u$ which does not induce a perfect matching in $N(u)$, since $w w^{\prime} \in M^{\prime}$ and $w \in N(u), w^{\prime} \notin N(u)$. This would contradict the fact that $G$ has the matched neighbors property. Thus $u w^{\prime} \in E$. By symmetry, it follows that it is enough to show $u^{\prime} w \notin E$, for concluding at the same time $u^{\prime} w \notin E$ and $u^{\prime} w^{\prime} \notin E$. Suppose that $u^{\prime} w \in E$. Then $M^{\prime}=\left(M \backslash\left\{u u^{\prime}, w w^{\prime}\right\}\right) \cup\left\{u w^{\prime}, u^{\prime} w\right\}$ is a perfect matching in $G-v$ which does not induce a perfect matching in $N(v)$, since for instance $u w^{\prime} \in M^{\prime}$ and $u \in N(v), w^{\prime} \notin N(v)$. Again, this contradicts the fact that $G$ has the matched neighbors property, and hence $u^{\prime} w, u^{\prime} w^{\prime} \notin E$. Next, consider the graph $G_{u}=G[\bar{N}+u]$. Let $M_{u}=M \cap E\left(G_{u}\right)$. We claim that for each vertex $y \in \bar{N}$ that belongs to the same connected component as $u$ in $G_{u}$, there exist two alternating paths with respect to $M_{u}$, one, say $P_{y}$, from $u$ to $y$ such that $y$ is incident with an edge of $M_{u}$ in $P_{y}$, and one, say $P_{y}^{\prime}$, from $u$ to $y$ such that $y$ is not incident with an edge of $M_{u}$ in $P_{y}^{\prime}$. This trivially holds for $w, w^{\prime}$. Now consider $y \in \bar{N}$ such thatwy $\in E$. Let $y^{\prime} \in \bar{N}$ such that $y y^{\prime} \in M_{u}$. First notice that $w y^{\prime} \in E$. Indeed, if $w y^{\prime} \notin E$, then $M^{\prime}=\left(M \backslash\left\{u u^{\prime}, w w^{\prime}\right\}\right) \cup\left\{v u^{\prime}, u w^{\prime}\right\}$ is a perfect matching in $G-w$ which does not induce a perfect matching in $N(w)$, since $y y^{\prime} \in M^{\prime}$ and $y \in N(w), y^{\prime} \notin N(w)$, a contradiction. Thus $w y^{\prime} \in E$. Now we get the desired paths: $P_{y}=u-w^{\prime}-w-y^{\prime}-y$ and $P_{y}^{\prime}=u-w^{\prime}-w-y$. Similarly we obtain $P_{y^{\prime}}=u-w^{\prime}-w-y-y^{\prime}$ and $P_{y^{\prime}}^{\prime}=u-w^{\prime}-w-y^{\prime}$. Thus the claim also holds for all vertices $y, y^{\prime} \in \bar{N}$ such that $y y^{\prime} \in M_{u}$ and at least one of $y, y^{\prime}$ is adjacent to some vertex in $\left\{w, w^{\prime}\right\}$. Now by repeating the above argument, we conclude that the claim holds.

Since $G$ has no cut-vertex, $G-u$ contains a path from $w$ to a vertex in $N(v)$. Let $y x$ be the first edge on this path, that goes from $\bar{N}$ to $N$. Hence $x \in N$, and $y \in \bar{N}$ is a vertex that is in the same connected component as $w$ in the graph $G[\bar{N}]$. If $x=u^{\prime}$, then consider the cycle $C=P_{y} \cup\left\{u^{\prime} y, u^{\prime} u\right\}$. Clearly $C$ is alternating with respect to $M$. Thus, by switching the edges of $C$, we obtain a perfect matching $M^{\prime}$ in $G-v$ such that $u^{\prime} y \in M^{\prime}$. Thus $M^{\prime}$ does not induce a perfect matching in $N$, a contradiction. So we may assume that $x \neq u^{\prime}$. Let $x^{\prime} \in N$ be such that $x x^{\prime} \in M$. Then $v-u^{\prime}-u-P_{y}$ is an alternating path from $v$ to $y$ with respect to $M$. By switching the edges of this alternating path, we obtain a perfect matching $M^{\prime}$ in $G-y$. Let $z \in \bar{N}$ such that $u z \in M^{\prime}$. As discussed in the first paragraph of the proof, every neighbor of $u$ in $\bar{N}$ is not connected to $u^{\prime}$; hence, $u^{\prime} z \notin E$. Since $M^{\prime}$ induces a perfect matching in $N(y)$, it follows that $y x^{\prime} \in E$. But now $M^{\prime \prime}=\left(M^{\prime} \backslash\left\{v u^{\prime}, x x^{\prime}\right\}\right) \cup\left\{v x, x^{\prime} y\right\}$ is a perfect matching in $G-u^{\prime}$ which does not induce a perfect matching in $N\left(u^{\prime}\right)$, since $u z \in M^{\prime \prime}$ and $u \in N\left(u^{\prime}\right), z \notin N\left(u^{\prime}\right)$, a contradiction.

The following result immediately follows from Propositions 3.10 and 3.11 .

Corollary 3.12. Let $G$ be a factor-critical graph satisfying the matched neighbors property. Let $\left\{B_{1}, B_{2}, \ldots, B_{q}\right\}$ be the block decomposition of $G$. Then each block $B_{i}, i=1,2, \ldots, q$, is an odd clique.
Using the above corollary, we now show that no partition allows to strongly co-satisfy all vertices in a factor-critical graph that satisfies the matched neighbors property. First let us consider the case when having a single block, i.e., $G$ is a clique of odd size.

Proposition 3.13. Let $G$ be a clique of odd size. Then any partition of $V$ (not necessarily balanced) strongly co-satisfies at most $(|V|-1) / 2$ vertices of $G$.

Proof. Observe that for any partition $V_{1}, V_{2}$ of $V$, all vertices in the larger set of the partition are not strongly co-satisfied.

Proposition 3.14. Let $G=(V, E)$ be a factor-critical graph satisfying the matched neighbors property. Then no partition $V_{1}, V_{2}$ of $V$ strongly co-satisfies all vertices in $\mathrm{V}$.

Proof. Consider a leaf block $B$ of $G$, and let $w$ be the cut-vertex corresponding to $B$. Let $V_{1}, V_{2}$ be a partition of $V$. By Proposition 3.13 the partition $V_{1}, V_{2}$ strongly co-satisfies at most $(|B|-1) / 2$ vertices of $B$ in the graph $G[B]$. Since every vertex $v \in B-w$ has no neighbors outside of $B$, the partition $V_{1}, V_{2}$ strongly co-satisfies $v$ in $G$ if and only if it strongly co-satisfies $v$ in $G[B]$. Hence at most $(|B|-1) / 2$ vertices in $B-w$ are strongly co-satisfied. Since $|B| \geqslant 3$, at least one vertex in $B-w$ is not strongly co-satisfied by $V_{1}, V_{2}$.

Even though Proposition 3.14 is sufficient for our purposes, we would like to highlight that the following stronger result holds as well.

Proposition 3.15. Let $G=(V, E)$ be a factor-critical graph satisfying the matched neighbors property. Then any partition of $V$ strongly cosatisfies at most $(|V|-1) / 2$ vertices.

Proof. We prove the result by induction on the number of blocks of $G$. Notice that in the proof of Proposition 3.10, we showed that if a graph $G$ is factor-critical satisfying the matched neighbors property and $B_{i}$ is a leaf-block of $G$ with corresponding cut-vertex $w$, then $G^{\prime}=G\left[\bigvee\left(B_{i}-w\right)\right]$ is factor-critical and satisfies the matched neighbors property. If there is just one block, then, by Proposition 3.12, $G$ is necessarily a clique of odd size and thus the result follows from Proposition 3.13. So we may assume that there exist at least two blocks. Let $V_{1}, V_{2}$ be a partition of $V$ and let $B$ be a leaf block of $G$. Let $w \in B$ be the (unique) cut-vertex of $G$ in $B$. Let $V^{\prime}=V \backslash(B-w)$. Recall that $B$ is a clique (see Proposition 3.12). We will distinguish two cases, depending on whether $\left|V_{1} \cap(B-w)\right| \neq\left|V_{2} \cap(B-w)\right|$ or $\left|V_{1} \cap(B-w)\right|=\left|V_{2} \cap(B-w)\right|$.

Case (a): $\left|V_{1} \cap(B-w)\right| \neq\left|V_{2} \cap(B-w)\right|$. Assume without loss of generality that $\left|V_{1} \cap(B-w)\right|>\left|V_{2} \cap(B-w)\right|$. Observe that all vertices in $V_{1} \cap(B-w)$ are not strongly co-satisfied with respect to $V_{1}, V_{2}$. Since $\left|V_{1} \cap(B-w)\right| \geqslant(|B|+1) / 2$, there are at most $(|B|-3) / 2$ vertices in $B-w$ that are strongly co-satisfied with respect to $V_{1}, V_{2}$. Furthermore, by induction, the partition $V_{1} \cap V^{\prime}, V_{2} \cap V^{\prime}$ strongly co-satisfies at most $\left(\left|V^{\prime}\right|-1\right) / 2$ vertices of $G\left[V^{\prime}\right]$. Notice that the vertex $w$ might be strongly co-satisfied with respect to $V_{1}, V_{2}$ in $G[V]$ but not with respect to $V_{1} \cap V^{\prime}$, $V_{2} \cap V^{\prime}$ in $G\left[V^{\prime}\right]$, since $w$ has neighbors in $B$. This implies that the partition $V_{1}, V_{2}$ strongly co-satisfies at most $\left(\left|V^{\prime}\right|-1\right) / 2+1$ vertices in $V^{\prime}$, where the +1 is due to the vertex $w$. Hence the total number of strongly co-satisfied vertices is at most $\left(\left|V^{\prime}\right|-1\right) / 2+1+(|B|-3) / 2=(|V|-1) / 2$.

Case (b): $\left|V_{1} \cap(B-w)\right|=\left|V_{2} \cap(B-w)\right|$. Then, exactly $(|B|-1) / 2$ vertices in $B-w$ are strongly co-satisfied. Furthermore, one can observe that the vertices in $G\left[V^{\prime}\right]$ that are strongly co-satisfied with respect to the partition $V_{1} \cap V^{\prime}, V_{2} \cap V^{\prime}$ are exactly the verti- 
ces that are strongly co-satisfied with respect to the partition $V_{1}$, $V_{2}$ in $G$. Thus, by induction, the total number of strongly co-satisfied vertices is at most $(|B|-1) / 2+\left(\left|V^{\prime}\right|-1\right) / 2=(|V|-1) / 2$.

Putting everything together, our 2-approximation for the MAX STRONG CO-SATISFYING BISECTION PROBLEM works as follows. In a first step we compute an Edmonds-Gallai decomposition $(X, Y, W)$ of the given graph $G=(V, E)$. If $Y \neq \emptyset$, we can apply Theorem 3.6 to find a bisection strongly co-satisfying half of all the vertices, which is obviously a 2-approximation.

Now assume $Y=\emptyset$. Notice that in this case, the graph $G$ consists of factor-critical odd connected components $G\left[X_{1}\right], \ldots, G\left[X_{p}\right]$ with $X_{1} \cup \cdots \cup X_{p}=X$, and of even connected components that admit a perfect matching and whose union is $G[W]$. Furthermore, since $G$ has an even number of vertices, we have that $p$ is even. Let $G\left[X_{1}\right], \ldots, G\left[X_{k}\right]$ be the factor-critical components satisfying the matched-neighbors property, and $G\left[X_{k+1}\right], \ldots, G\left[X_{p}\right]$ be those that do not satisfy the matched neighbors property. For each $i \in\{1, \ldots, k\}$, we apply Proposition 3.9 to find a partition $X_{i, 1}, X_{i, 2}$ of $X_{i}$ with $\left|X_{i, 1}\right|=\left|X_{i, 2}\right|+1$ that strongly co-satisfies at least $\left(\left|X_{i}\right|-1\right) / 2$ vertices of $X_{i}$. Furthermore, for $i \in\{k+1, \ldots, p\}$ we use Proposition 3.8 to find a partition $X_{i, 1}, X_{i, 2}$ of $X_{i}$ with $\left|X_{i, 1}\right|=\left|X_{i, 2}\right|+1$ that strongly co-satisfies at least $\left(\left|X_{i}\right|+1\right) / 2$ vertices of $X_{i}$. Additionally, since $G[W]$ admits a perfect matching, we can apply Theorem 3.5 to find a bisection $W_{1}, W_{2}$ of $W$ that strongly co-satisfies at least $|W| / 2$ vertices of $W$. We finally return the bisection $V_{1}, V_{2}$ given by

$$
\begin{aligned}
& V_{1}=W_{1} \cup \bigcup_{i=1}^{p / 2} X_{i, 1} \cup \bigcup_{i=p / 2+1}^{p} X_{i, 2}, \\
& V_{2}=W_{2} \cup \bigcup_{i=1}^{p / 2} X_{i, 2} \cup \bigcup_{i=p / 2+1}^{p} X_{i, 1} .
\end{aligned}
$$

First, it is easy to check that $V_{1}, V_{2}$ is indeed a bisection, i.e., $\left|V_{1}\right|=\left|V_{2}\right|$. That the partition can be found in polynomial time follows from the fact that all results used in the above discussion are constructive and come with efficient algorithms. Furthermore, by the above discussion the total number of vertices being strongly co-satisfied by $V_{1}, V_{2}$ is at least

$\sum_{i=1}^{k}\left(\left|X_{i}\right|-1\right) / 2+\sum_{i=k+1}^{p}\left(\left|X_{i}\right|+1\right) / 2+|W| / 2 \geqslant(|V|-k) / 2$.
By Proposition 3.14, we have that any partition $V_{1}^{\prime}, V_{2}^{\prime}$ of $V$, satisfies at most $\left|X_{i}\right|-1$ vertices in $G\left[X_{i}\right]$ for $i \in\{1, \ldots, k\}$. Hence, no partition - not even one that is not a bisection - can strongly co-satisfy more than $|V|-k$ vertices of $G$. Together with (2), this implies that the proposed algorithm is indeed a 2-approximation for finding a bisection maximizing the number of strongly co-satisfied vertices. More precisely, it shows that there is not even a partition of $V$ that strongly co-satisfies strictly more than twice the number of vertices that are strongly co-satisfied by the proposed algorithm.

\section{Conclusion}

In this paper we considered the MAX SATISFYING BISECTION PROBLEM and presented a polynomial-time 2-approximation algorithm. This answers an open question from [3]. It remains open whether one can do better, i.e., whether there exists a polynomial-time $c$-approximation algorithm for $c<2$.

\section{Acknowledgements}

This paper was written when the first author was an Assistant Professor in the Centre for Discrete Mathematics and its Applications (DIMAP) at the University of Warwick. The support of the institution is gratefully acknowledged.

\section{References}

[1] C. Bazgan, Z. Tuza, D. Vanderpooten, The satisfactory partition problem, Discrete Applied Mathematics (154) (2006) 1236-1245.

[2] C. Bazgan, Z. Tuza, D. Vanderpooten, Approximation of satisfactory bisection problems, Journal of Computer and System Sciences 75 (5) (2008) $875-883$.

[3] C. Bazgan, Z. Tuza, D. Vanderpooten, Satisfactory graph partition, variants, and generalizations, European Journal of Operational Research 206 (2) (2010) 271-280.

[4] M. Gerber, D. Kobler, Partitioning a graph to satisfy all vertices, Technical Report, Swiss Federal Institute of technology, Lausanne, 1998.

[5] M. Gerber, D. Kobler, Algorithmic approach to the satisfactory graph partitioning problem, European Journal of Operational Research (125) (2000) 283-291.

[6] A. Schrijver, Combinatorial Optimization, Polyhedra and Efficiency, Springer, 2003.

[7] V.V. Vazirani, Approximation Algorithms, Springer, 2003.

[8] D.B. West, Introduction to Graph Theory, Prentice Hall, 2001 